\title{
VARIA
}

\section{LE STÉRÉOTYPAGE MÉDIATIQUE DU GENRE FÉMININ}

\author{
Du procès d'aliénation à l'introduction \\ d'une mascarade stratégique
}

\begin{abstract}
Justine Marillonnet ${ }^{1}$
Cet article a pour vocation d'ouvrir une nouvelle perspective quant aux fonctions du procédé de stéréotypage dans les messages médiatiques à l'adresse d'un public féminin. Mobilisant la sémiologie des indices pour produire une étude en production des images de mode présentées dans trois titres de presse magazine féminine française, cette analyse tend à introduire le rôle d'amorce du stéréotype dans le contrat de lectorat et propose son utilisation stratégique, non plus pour une énonciation aliénante quant aux représentations du genre mais pour une négociation du genre mettant en scène une nouvelle forme de mascarade.
\end{abstract}

Aujourd'hui, tandis que la presse magazine féminine est consommée par plusieurs millions de femmes en France, comment prétendre à une aliénation du public par un discours stéréotypé performatif ? Admettons la présence du stéréotype de genre, démontrée déjà dans de nombreuses analyses de cette presse (Soulages, 2004 ; Chabrol, 2007) : comment

1 Docteure en Sciences de l'Information et de la Communication, Université Lyon 2, France.

Recherches en communication, $\mathrm{n}^{\circ} 36$ (2011). 
postuler que ce discours maintient les femmes dans une soumission aux normes androcentrées de notre société contemporaine, sans laisser entendre que le public féminin est une foule irréfléchie, inactive lors de la réception d'un message médiatique?

Considérant le projet d'identification de la cible dans le contrat de lecture (Charaudeau, 1983 ; Veron, 1985), il est intéressant de s'interroger sur les fonctions du stéréotype dans un tel dessein de reconnaissance des cibles. Le public féminin, pouvant exercer son droit de retrait, ne pourrait se contenter d'être ce public passif, soumis aux injonctions des médias. Si l'identité du lectorat, de façon indicielle, est contenue et traduite dans les discours et images du média, comment poursuivre ce procès d'aliénation de la presse magazine à l'adresse du public féminin ciblé ?

Le contrat de lecture prévoit, pour le succès du processus de communication (avec un double objectif économique et sémiologique), un partage de valeurs entre les deux instances en présence (énonciatrice et réceptrice) ainsi qu'une reconnaissance mutuelle de leur identité. Donc, si stéréotype il y a, ne peut-on pas chercher désormais d'autres fonctions à ce dernier, plus vraisemblables aujourd'hui que la simple fonction de reproduction des normes ? Devant l'enjeu de captation qui anime les médias, le stéréotype n'a-t-il pas un rôle à remplir?

L'analyse de la publicité a étudié le processus de stéréotypage et démontré la permanence de stéréotypes de genre, à l'adresse des consommateurs (Goffman, 1977 ; Soulages, 2004). Toutefois, ce constat n'est pas à associer directement à celui d'une étude de la presse magazine féminine. En effet, le procédé de stéréotypage dans les messages publicitaires prend fonction de raccourci cognitif efficace pour la reconnaissance des cibles de consommateurs et s'apparente davantage à un consensus dans la communication qu'à un véritable partage de valeurs : les discours publicitaires privilégient encore quantitativement des imaginaires de genre consensuels et tendent à promouvoir majoritairement des représentations du masculin et du féminin satellisées autour de valeurs patriarcales traditionnelles (Pahud, 2009). Mais le cas de la publicité est différent de celui de la presse magazine, ces deux supports n'obéissant pas à la même logique. Le dispositif médiatique de la presse magazine féminine doit répondre à une double logique, certes économique mais aussi sémiologique, là où la publicité a principalement une visée commerciale et doit être efficace pour un public large, mixte, hétérogène. La publicité est diffusée auprès d'une cible, de façon unilatérale, sans que celle-ci ne procède à une sélection des messages. 
La presse magazine, elle, est un système de signes consommé et choisi par les lectrices, après acceptation du contrat de lecture instaurant la relation symbolique entre elles et ce support.

L'analyse de la littérature a permis de dresser un autre constat quant au procédé de stéréotypage. En effet, l'étude approfondie de la littérature, traitant des héroïnes romanesques du passé (Heinich, 1996) ou des fictions et autofictions d'auteures féminines (Détrez, 2004, p. 32), démontre une forme d'ambivalence dans les représentations, et interroge le contre-stéréotype féminin : « La simple inversion des modèles est-elle garante de leur affranchissement, ou, plus subtilement, ne contribue-t-elle pas à en maintenir l'actualité ? » (Détrez, 2004). On peut instruire la question de l'emploi du contre-stéréotype dans la représentation du genre féminin, en y voyant l'activation première du stéréotype, avant l'introduction du contre-stéréotype. Là encore, nous restons face au constat de procédés de stéréotypage ou de contre-stéréotypage ayant pour résultante la représentation du genre féminin dans un maintien des normes et des rôles genrés traditionnels.

Le discours de la presse magazine, les messages publicitaires, les parcours de vie des héroïnes de la littérature : l'ensemble de ces supports convoquent une représentation stéréotypée du genre féminin faisant admettre certains procès féministes à leur égard. Toutefois, le cas de la presse féminine n'a pas été pleinement traité, les images de mode ne figurant à ma connaissance dans aucune étude de dispositifs médiatiques du champ des sciences de l'information et de la communication. Néanmoins, on note que la psychosociologie s'est intéressée à ce cas particulier (Chabrol, 2007), par l'analyse de la performativité des stéréotypes dans la presse magazine féminine. Il ressort de ces études que seuls les comportements déjà stéréotypés du lectorat sont renforcés par la présentation du stéréotype médiatique. Ainsi, comme de précédentes théories des effets limités des médias ont pu le démontrer, le stéréotype ne fonctionnerait pas, dans la presse magazine féminine, comme une prescription systématiquement efficace.

La consommation, organisée par ce quadrillage expansionniste [les réseaux des médias], ferait figure d'activité moutonnière, progressivement immobilisée et " traitée 》 grâce à la mobilité croissante des conquérants de l'espace que sont les médias. Fixation des consommateurs et circulation des médias. Aux foules, il resterait seulement la liberté de brouter la ration de 
simulacres que le système distribue à chacun. Voilà précisément l'idée contre laquelle je m'élève : pareille représentation des consommateurs n'est pas recevable. (De Certeau, 1990, p. 240)

Cette citation contribue à me faire admettre la possibilité d'une autre fonction pour le stéréotype médiatique adressé au public féminin. Se réappropriant le discours médiatique au cours d'une interprétation personnelle des messages, le public pourrait déployer un effort de compréhension plus complexe qu'il n'y paraît autour des différentes constructions de représentations de l'identité féminine.

Le choix de l'étude des images de mode de la presse magazine féminine s'impose, outre le fait qu'elle n'a jamais fait l'objet d'une étude à part entière et qu'en cela déjà, l'étude sera d'une certaine manière, originale. L'image de mode convoque les langages du corps et de la mode dans une mise en scène soumise à un contrat de lecture. L'image, en tant qu'outil de composition de nouveaux mondes fictionnels possibles (Goodman, 1992), parfois insaisissables par le discours, répond également à un projet narratif particulier. L'image de mode, comme l'image artistique, n'est pas nécessairement représentative. Elle demeure pour autant un indicateur du social intéressant pour nos études, en ce qu'elle représente, non pas une réalité vécue, mais l'imaginaire socio-médiatique de la société qui la produit. Aussi, dans la mesure où, comme Monneyron, nous caractérisons la mode comme un concept et un phénomène social liés à l'avènement des sociétés fondées sur l'individu, alors nous pouvons consacrer la mode et la production d'images de mode au rang d'indicateurs sociaux.

Si la photographie, comme la psychanalyse, nous permet de plonger dans les profondeurs psychiques, elle nous permet aussi de plonger dans les profondeurs sociétales. [...] c'est que, à travers elle, peuvent se lire les mythes, les images et les symboles, et, par suite, les grandes structures anthropologiques qui définissent une époque et lui donne un sens. Elle est mieux, même, que le simple révélateur d'un inconscient collectif. (Monneyron, 2010, p. 155)

Si nous ajoutons à cela l'intérêt de l'analyse d'une image en tant que vecteur de nouvelles connaissances (Fleury, 2006), alors nous trouvons dans l'analyse des images de mode de la presse féminine un terrain privilégié pour l'analyse des représentations du genre féminin, 
les considérant représentations sociales innovantes et éléments de nouvelles connaissances.

Enfin, la mode, plus qu'un prétexte à la mise en scène, n'est pas un objet frivole ici. Il nous faut considérer l'objet vêtement en tant que « médium », en tant que prolongement, ici, du féminin (Bohn, 2001). L'image de mode, médiatisée par la presse magazine féminine, est un objet dans lequel se précipitent les imaginaires sociaux, les codes esthétisants d'une démarche photographique artistique et les désirs identificatoires du public féminin à travers les choix de vêtements. En cela, l'image de mode et les stéréotypes qu'elle peut mettre en scène, sont des représentations de l'imaginaire féminin co-construites (par les médias et par leurs publics), des mondes possibles fictionnels désirables (Semprini, 2000) dans lesquels les femmes se projettent. Ces systèmes de signes (corps, vêtement, image) évoquent, au-delà du social, des hypothèses de réalisation pour les sujets féminins ciblés. Le stéréotypage de genre ne peut, dans une telle mesure, répondre seulement au nivellement des perspectives identitaires du public féminin, sa présence au sein d'un contrat de lecture alimente d'autres objectifs qu'il nous reste à identifier.

\section{La méthodologie : l'analyse des indices pour l'étude d'une représentation ${ }^{1}$}

L'analyse des images souffre d'une impossible automatisation du travail de recherche. Si des logiciels permettent l'analyse automatique d'un discours, garantie d'un travail objectif, il n'existe pas d'outils performants pour dresser des typologies d'images, où l'œil du chercheur ne soit investi à titre personnel. Il reste essentiel de fournir une grille d'analyse pour cette analyse sémiologique des représentations, n'obéissant pas à un jugement de valeurs quant au bienfondé de représenter les femmes de telle ou telle autre manière. Cette grille devra permettre une première phase d'analyse structurale pour présenter une typologie des mises en scène du genre féminin. Par la suite, une phase d'analyse interprétative des indices ainsi répertoriés, convoquant des interprétants externes, apportera la possibilité de se placer dans un schème fonctionnel pour saisir le nouveau rôle du stéréotype. Si l'étude procède d'abord d'une analyse isolée de chacune des images dans la

1 (Houdebine, 2004) 
phase structurale, c'est bien ensuite à l'échelle de l'ensemble des séries mode présentes que l'analyse interprétative pourra se révéler efficace.

Avant d'élaborer ces outils d'analyse, il est nécessaire de travailler en immanence sur le corpus et d'étudier un premier échantillon d'images de mode. Après cette phase d'exploration, associée à une recherche documentaire quant à de précédentes typologies de représentations du genre féminin, un premier répertoire d'indicateurs et de marqueurs à identifier est réalisé. La difficulté de cette analyse réside aussi dans la définition même de ce qui relève ou non du stéréotype de genre. Pour instruire cette question, plusieurs typologies émises lors d'études sur la figuration des sujets féminins (Kaufmann, 1995, 2005) ou encore sur la représentation médiatique du genre féminin (Goffman, 1977 ; Soulages, 2009) sont confrontées. Concernant la reconnaissance d'un stéréotype dans une mise en scène, les conseils aux lecteurs de Goffman sont précieux :

[...] il suffit pour prendre aussitôt conscience du stéréotype, d'imaginer pour chaque photographie ce qui résulterait si les sexes étaient échangés. Avec cette possibilité à l'esprit, le lecteur sera en mesure de produire ses propres commentaires et pourra se faire une idée des mérites éventuels des miens. (Goffman, 1977, p. 34)

C'est ainsi du croisement de ces données empiriques, théoriques et méthodologiques que j'élabore une grille d'analyse reprenant chacun des indicateurs dont la présence est à mesurer au sein du corpus : indicateurs du corps, du vêtement et des décors. Une série d'indicateurs est définie comme codant pour les mises en scène sociales, une autre pour les mises en scène intimes.

Le corpus est ensuite finalisé, et le choix d'une année de parutions de séries mode dans la presse magazine féminine semble assurer les propriétés de signifiance, d'acceptabilité et d'exploitabilité d'un corpus. Cette étude contemporaine se base ainsi sur l'année 2008 et désigne trois titres de presse magazine féminine : Femme Actuelle, ELLE et Cosmopolitan. Le choix de ces titres relève d'un effort de caractérisation de l'offre sur le marché de la presse magazine féminine. Ils appartiennent à trois groupes de presse différents, parmi eux figurent deux hebdomadaires et un mensuel, et répondent à trois ciblages différents pour leurs lectorats. 


\begin{tabular}{|c|c|c|c|c|}
\hline & $\begin{array}{l}\text { Groupe de } \\
\text { presse }\end{array}$ & Périodicité & Profil du lectorat ${ }^{1}$ & $\begin{array}{l}\text { Ventes } \\
\text { moyennes }^{2}\end{array}$ \\
\hline $\begin{array}{l}\text { Femme } \\
\text { Actuelle }\end{array}$ & $\begin{array}{l}\text { Groupe Prisma } \\
\text { Presse }\end{array}$ & hebdomadaire & $\begin{array}{l}46,9 \% \text { du lectorat a plus } \\
\text { de } 50 \text { ans }\end{array}$ & $\begin{array}{l}927420 / \\
\text { semaine }\end{array}$ \\
\hline $\begin{array}{l}\text { Cosmopolitan } \\
\text { France }\end{array}$ & $\begin{array}{l}\text { Groupe } \\
\text { Marie-Claire }\end{array}$ & mensuel & $\begin{array}{l}60 \% \text { du lectorat a moins } \\
\text { de } 35 \text { ans }\end{array}$ & $377884 /$ mois \\
\hline Elle France & $\begin{array}{l}\text { Groupe } \\
\text { Lagardère }\end{array}$ & hebdomadaire & $\begin{array}{l}66 \% \text { du lectorat à moins } \\
\text { de } 50 \text { ans } \\
37 \% \text { a moins de } 35 \text { ans }\end{array}$ & $\begin{array}{l}370658 / \\
\text { semaine }\end{array}$ \\
\hline
\end{tabular}

Tableau 1 : Corpus

Basés sur 482 images de mode réparties sur 12 numéros de Cosmopolitan, 12 numéros de ELLE et 12 numéros de Femme Actuelle (choisis de façon aléatoire sur l'année afin d'équilibrer la répartition des séries à étudier avec le mensuel Cosmopolitan), mes travaux rendent compte d'une utilisation systématique du stéréotype de genre, à l'instar des travaux sur les messages publicitaires et sur les portraits d'héroïnes littéraires. Le procédé de stéréotypage est identifié dans cette recherche par la présence plus ou moins importante des marqueurs du contexte, du corps et du vêtement, codant pour l'une ou pour l'autre des deux mises en scènes récurrentes et définies comme stéréotypées au préalable. La découverte ici ne tient pas à la reconnaissance du stéréotype, mais bien à l'apparition d'une catégorie résiduelle de mises en scène, ne faisant pas référence aux indicateurs usuellement convoqués pour le stéréotype, lequel n'apparaît donc pas autonome dans cette représentation du genre féminin mais vraisemblablement complémentaire d'autres processus de représentation. De ce travail, articulé sous la forme d'un work in progress, émerge cette nouvelle catégorie de représentations, non pas isolées au sein du corpus, mais organisées autour de nombreuses régularités. Les mises en scène sont essentiellement repérables en fonction des marqueurs du contexte, qui dictent ici l'effort de typologie. Les mises en scène sociales sont présentées dans des espaces publics, souvent à l'extérieur tandis que les mises en scène intimes relèvent

1 Sources : groupes de presse et AEPM : Audience et Etudes de Presse Magazine. L'organisme dont la finalité est de mesurer et de qualifier les audiences de la presse magazine réalise deux fois par an une étude d'audience par entretien à domicile des principaux magazines français (plus de 100000 lecteurs).

2 Ventes moyennes selon la périodicité. Source : OJD 2009. Office de Justification de la Diffusion. L'organisme contrôle les tirages et la diffusion de la presse dans le monde. 
d'un cadre intérieur, jour ou nuit (souvent l'intimité d'une chambre), les nouvelles mises en scène, elles, sont « hors-contexte».

C'est à l'issue de cette exploration particulière du sujet, mobilisant déjà une première phase d'analyse, que les hypothèses de recherche s'imposent. Il s'agit d'abord de confirmer la possibilité d'une représentation complexe du genre féminin, malgré le recours aux stéréotypes de genre. Cette représentation dans les images de mode serait apte à signifier la pluralité de possibilités d'expression du genre féminin et ferait écho au morcellement identitaire de l'identité féminine, déjà pressenti dans les précédentes études instruisant la question d' " un » genre féminin. Ces nouvelles représentations répondraient aux besoins de reconnaissance et d'identification des cibles féminines, et à un nouvel imaginaire, propre au monde des femmes, dans lequel ces dernières projetteraient des désirs identificatoires spécifiques. Tenant compte de l'impossible soumission totale des publics aux diktats d'un message médiatique aliénant, l'on s'interroge sur un éventuel rôle joué par le stéréotype dans l'énonciation médiatique, qui ne se poserait pas en termes de performativité pour le maintien de «l'ordre des choses » (Bourdieu, 1998), mais davantage en termes d'amorce, dans le contrat de lecture passé entre les instances productrice et énonciatrice (Charaudeau, 2000).

\section{Nouvelle typologie des représentations du genre féminin}

La typologie des images de mode étudiées, fruit de l'analyse des indices dans les mises en scène, permet le constat suivant : deux catégories de mises en scène de femmes dans la mode proposent une vision stéréotypée du genre féminin, une catégorie résiduelle d'images interroge la place du stéréotype situé en amont.

Les mises en scène stéréotypées placent les modèles féminins dans des situations sociales variées, et présentent des utilisations du corps et du vêtement plus ou moins adaptées à l'action. Par exemple, Femme Actuelle propose des mises en scènes ancrées dans la sphère sociale publique, les femmes sont souvent à l'extérieur, dans la rue, dans des actions identifiables simples (sortie du bureau, avant le dîner en ville, etc). Le corps est peu mis en avant, les vêtements sont adaptés au contexte, il apparaît qu'un effort en termes de représentativité soit effectué ici. ELLE, à l'opposé, propose des mises en scène dans la sphère publique et dans la sphère intime, qui toutes deux font appel à une mise à disposition du corps de la femme, dans la séduction, signifiée ici par des postures d'auto-contact récurrentes, des 
bouches entrouvertes, des regards pénétrants selon un axe Y-Y (« yeux dans les yeux » face à l'objectif) ou encore un regard mi-clos, signe de rêverie ou d'abandon. Cette séduction est également mise en scène dans le vêtement, qui déshabille plus qu'il n'habille le modèle féminin (nombreux corps dénudés durant des scènes à l'extérieur, dans l'espace public). Les mises en scène sociales dans ELLE permettent d'identifier une femme dans la rue (contexte public) mais alanguie sur les marches d'une ruelle, le corsage ouvert et les jambes écartées, la tête en arrière, bouche entrouverte, en posture d'auto-contact. Les mises en scène de l'intime proposent quant à elles l'incarnation de fantasmes masculins (la série mode intitulée « Belle de jour », en référence au film de Luis Buñuel, en est un cas exemplaire).

Le cas de Cosmopolitan est tout à fait remarquable, tant le stéréotype y est prononcé. Les femmes mises en scène dans la sphère publique, sous le regard de la société, se muent en caricatures de l'oisive, tandis que les femmes mises en scène dans la sphère privée deviennent les vamps, caricatures d'une séduction charnelle. C'est le seul magazine à présenter systématiquement dans chaque titre, une série mode dans laquelle le modèle féminin est totalement soustrait aux sphères publique et intime (aucun décor ni action). Attendant dans ce cas une absence de postures et de parades de genre (puisque ni le regard de la société ni celui de l'homme ne sont parties prenantes), le lectorat peut se laisser surprendre par une utilisation du corps et du vêtement particulièrement détonante. Cette catégorie résiduelle de mises en scènes montre des modèles féminins désarticulés, des corps qui ne séduisent plus, « anti naturels », déstructurés : les pieds ne touchent plus le sol, les bras et les mains sont en l'air, les visages se font grimaçants. Quelques images, réparties au sein de ces séries, ponctuent cette apparente dénaturalisation du corps féminin, par une réappropriation paroxysmique des parades genrées.

ELLE ne reprend jamais au sein de ce corpus ce type de mises en scène hors contexte. Femme Actuelle tente une mise en scène assimilable par quelques points à celle de Cosmopolitan, mais sans subversion pour les postures du corps : les normes de genre ne disparaissent pas, les corps et les vêtements obéissent aux règles de bienséance traditionnelles pour la figuration féminine, seul l'effort de soustraction au contexte social et intime apparaît dans l'absence de décors. La tentative d'extraire le personnage féminin du jeu social ou de la séduction est amorcée dans quelques séries de Femme Actuelle, mais le personnage demeure fidèle aux représentations normatives du genre féminin. 
Si les deux premiers titres, ELLE et Femme Actuelle, convoquent le stéréotype au service de la mise en scène traditionnelle de parades de genre (entre parades sociales et nécessaire séduction féminine), Cosmopolitan ne convoque plus seulement la parade mais plutôt la parodie de parades pour le déploiement du genre. Une nouvelle identité féminine, longtemps contrainte à envisager le corps comme seul lieu naturel d'expression, reprend aujourd'hui ces codes normatifs pour son propre compte et semble sur-jouer, consciemment, les parades de genre.

Cette phase d'analyse structurale permet donc d'établir trois catégories de mises en scène du genre : deux font pleinement écho au stéréotype, une dernière, typique du dispositif de Cosmopolitan (elle se retrouve dans chacun des titres du corpus - les 12 titres de l'année 2008) contourne les normes. L'analyse interprétative permet alors de saisir les enjeux d'un tel dispositif de représentation du genre féminin et d'envisager de nouvelles fonctions pour le procédé de stéréotypage. À la première hypothèse, la vérification suivante est proposée : la représentation du genre féminin dans les images de mode de la presse magazine française étudiée fait écho à une pluralité de représentations possibles. La définition d'une féminité en tant que version universelle et figée du féminin n'est pas l'objet de l'énonciation des magazines de mode étudiés. En outre, si ELLE ou Femme Actuelle utilisent une représentation exclusive des femmes, sociale ou intime, ces supports, fonctionnant dans le système plus vaste « magazines de mode », ne font que répondre à un segment de public, laissant ce dernier libre dans son choix de consommation de modèles médiatiques. L'offre en termes de représentations du genre féminin dans la presse magazine féminine est plurielle, à l'image du morcellement de l'identité féminine et de son ambivalence. Pour reprendre le célèbre axiome lacanien, « la femme n'existe pas », mais « les femmes existent».

Étant admis que le stéréotype ne fonctionne pas seul, dans l'ensemble du corpus et au sein même du titre Cosmopolitan, il est intéressant de mettre en perspective ce procédé et les nouvelles représentations émergentes - la caricature de parades et la soustraction du personnage féminin à l'espace social.

\section{Fonction stratégique du stéréotype dans l'énonciation}

Les deux titres, ELLE et Femme Actuelle, ont tous deux recours au procédé de stéréotypage, mais l'utilisent à des fins sémiologiques bien distinctes. Femme Actuelle convoque le stéréotype dans une mise 
en scène sociale, favorisant une reconnaissance du public féminin dans cette identité sociale normative, peu encline à la séduction ou à l'érotisation, sans doute en rapport avec sa cible de plus de 50 ans (on pourrait encore s'interroger sur cette absence de séduction pour ces femmes) ; tandis que ELLE le mobilise dans un jeu de séduction permanent, mettant en scène, non plus seulement le regard de la société, mais le regard de l'homme, dans des mises en scène de l'intime débordant sur la sphère sociale. La pluralité des possibilités représentationnelles reste assurée, malgré le recours au stéréotypage.

Lorsque le stéréotype est central dans la représentation du genre féminin (à la manière de ELLE par exemple), la conclusion la moins hâtive que l'on puisse fournir, avant de procéder à une étude en réception, consiste à supposer la souscription du lectorat à ce procédé dans le cadre d'un contrat de lecture. L'injonction normative, s'il en est, ne doit pas seulement alimenter le procès de la presse féminine, mais doit être comprise dans un système plus complexe, faisant appel aux théories psychologiques de réception des messages médiatiques. Les publics, récepteurs actifs (dans le processus de signification des messages médiatiques) et prescripteurs (en tant que clients des médias) des contenus et des dispositifs médiatiques, participent du succès économique et sémiologique des médias.

Dans le cas d'une utilisation complémentaire du stéréotype au sein d'un titre, on constate que ce dernier est présenté en amont de représentations hors contexte. Ce processus est engagé en tant qu'élément du dispositif médiatique et non en tant que finalité dans la représentation du genre féminin. Si le stéréotype s'avère performatif ici, c'est que son activation accroit la reconnaissance de la cible féminine, non pas dans une perspective d'intériorisation inconsciente des normes genrées mais dans un projet d'amorce pour de nouvelles représentations. Le cas de Cosmopolitan illustre parfaitement cette proposition.

Tantôt utilisé pour sa propre caricature, tantôt utilisé en amorce de représentations contournant les normes, le stéréotype semble prescrit ici, dans un sur-jeu d'actrices en interaction. Le lien entre prescription du stéréotype et prescription du symptôme se tisse, propice à une nouvelle négociation des ambivalences du genre féminin : "Un symptôme se forme à titre de substitution à la place de quelque chose qui n'a pas réussi à se manifester au dehors. » (Freud, 2004, p. 303). « Guérir le mal par le mal », être dans une monstration des parodies de genre, pourrait permettre une ouverture, un défigement de la mascarade subie (Rivière, 1929), au profit d'une mascarade stratégique. En effet, s'il est question 
de mascarade dans l'utilisation des parades dans la figuration féminine, cette dernière a pour vocation de masquer le «non-masculin », de jouer la féminité, à des fins identificatoires parfois bien minces, confinant davantage à l'excuse de l'existence qu'à sa revendication. Ici, le stéréotype, mis en scène par les langages du corps et du vêtement, devient un moyen dans la figuration des personnages féminins et non plus une fin. Ce moyen, basé sur la prescription de normes genrées imposées par une société androcentrée, est un outil spécifique dans le cadre de l'identification des sujets féminins, puisqu'il fait l'objet d'un détournement. C'est de ce détournement conscient (car caricatural) des parades figuratives de genre, qu'on peut entrevoir les contours d'un nouveau projet d'identification du genre à travers une mascarade stratégique.

Le vêtement est [...] une question beaucoup plus féminine que masculine, comme en témoigne ne serait-ce que l'embarras du choix existant en matière de tenues pour femmes, ou la teneur des conversations [...] cette dimension féminine de la question vestimentaire est l'indice d'une sensibilité particulière à une condition identitaire plus difficile, plus problématique, plus délicate à gérer pour les femmes qu'elle ne l'est pour les hommes. (Heinich, 1996, p. 334)

Cette proposition se lit en complément des travaux sur la société de loisirs, dans lesquels est présenté le statut de la femme en tant que traduction du statut du père, du frère ou de l'époux, ne permettant aucune subjectivité pour le féminin passé sous silence (Veblen, 1978). Utiliser les langages du corps et de la mode n'est donc pas neutre dans la mascarade stratégique. La figuration des femmes, résumée par le passé à des efforts d'ostentation en termes de parures et de postures, afin de signifier à autrui le niveau de richesse de l'homme référent, est aujourd'hui négociée pour en faire un moyen d'identification du sujet féminin pour un public féminin. Maintenant ainsi l'ordre des choses et poursuivant la figuration féminine telle que précédemment prescrite, les publics féminins choisissent de se reconnaître dans des versions stéréotypées du genre féminin, si ces dernières sont associées à des versions innovantes du genre. Maîtrisant pour son compte les parades de genre, la femme mise en scène dans ces images « hors contexte » prétend à un contournement voire une subversion des normes. La prescription du stéréotype, en tant que symptôme (Watzlawick, 1972), a donc amené le modèle féminin à dépasser les parades de genre et à proposer, à partir de ce même langage, une nouvelle parole, celle du sujet féminin. 
Le symptôme de la féminité (le stéréotype), mis à la place de ce qui ne devait pas exister (le sujet féminin), une fois prescrit, contribue ainsi à redéfinir les comportements du sujet. Le genre féminin, pluriel, mobile, s'adapte à un imaginaire social lui-même en perpétuelle évolution et répond aux transformations de l'environnement par le biais d'un remaniement de ses conditions d'identification. Comme toute représentation sociale, et médiatique, celle du genre féminin peut évoluer par une « intégration de nouvelles données » (Moscovici, 1976), qu’elles soient sociales ou imaginaires, et par la performance de nouvelles valeurs du genre, mises en avant dans l'image de mode, créative, artistique, dépourvue d'une visée vériste de la réalité.

Le procédé de stéréotypage prend fonction d'une prescription du symptôme, dans ce cadre précis de l'imaginaire de la mode. En effet, ce défigement des normes du genre féminin est permis et interprétable dans le monde des femmes (Touraine, 2006) et dans notre cas, dans celui de la mode. La mode, en tant que langage du féminin, dépasse une simple fonction d'ornementation pour revêtir la fonction d'outil, au service, non plus de « La Femme » ou d'un éternel féminin, mais de l'expression des sujets féminins.

\section{Conclusions et nouvelles perspectives}

Considérant le caractère, non plus autonome et performatif du stéréotype dans les images de mode de la presse magazine féminine, mais complémentaire et stratégique dans l'effort de représentations des sujets féminins, il est possible de mieux comprendre sa permanence au sein des contrats de lecture de la presse magazine féminine. Tantôt outil du consensus dans le travail de reconnaissance des publics, tantôt amorce pour le contournement des normes, le stéréotype assure l'établissement d'un code partagé entre les médias et leurs publics. Ce procédé ne peut être compris indépendamment du système de représentations dans lequel il évolue. Pris en tant qu'élément dans un système de signes plus vaste, celui de la mode dans la presse magazine féminine, la question de sa fonction peut être instruite, sans alimenter davantage la condamnation de la presse féminine en tant qu'outil d'aliénation. Au-delà même de cette déconstruction de la condamnation, j'ai supposé la fonction stratégique du stéréotype, non pas dans la logique économique des magazines, mais dans la visée sémiologique parfois émancipatrice de la presse féminine. Contournant les normes de genre et négociant les parades dans de nouvelles mises en scène extraites de tout champ pres- 
criptif, les images de mode permettent d'introduire de nouvelles représentations du genre féminin, sur-jouant la féminité jusqu'à prescrire de nouveaux comportements inattendus, subversifs, et pourquoi pas anticipateurs. L'usage du stéréotype ouvre un nouveau champ de possibles en termes de représentations d'abord, puis de comportements peut-être. Conscientes de la parade, les femmes peuvent interpréter la complexité de cette démarche stratégique de mascarade, les amenant à maitriser le langage du corps et du vêtement, cette fois pour leur propre compte.

Une modification de l'imaginaire social, une modification du système de représentations des relations de genre, peut induire, sur le long terme, sans nécessaire rupture ou révolution, un changement de pratiques. Aujourd'hui, ces nouvelles représentations du genre féminin sont introduites dans le monde des femmes, mais ne peut-on pas supposer qu'elles soient relayées au sein de la société et indiquent alors de nouveaux comportements en termes de figuration ? Ce type d'études, en réception, demande une association plus étroite avec le champ de la psychologie sociale et nécessite d'approfondir le moment de la réception et de l'interprétation de ces représentations médiatiques auprès des publics, féminins et masculins. Si l'univers des images de mode de la presse magazine relaie auprès des femmes ces nouvelles possibilités de production du genre féminin, il nous reste à instruire la question de l'acceptation de ce monde fictionnel possible pour l'ensemble de la société.

\section{Références}

Bohn, C. (2001). Le vêtement comme medium. Dans F. Monneyron, Le vêtement. (pp. 189-204). Paris : L'Harmattan.

Bourdieu, P. (1998). La Domination masculine. Paris : Seuil.

Chabrol, C. (2007). Catégorisation de genre et stéréotypage médiatique : du procès des médias aux processus socio-médiatiques. Dans H. Boyer, Stéréotypage, stéréotypes, fonctionnements ordinaires et mises en scène (pp. 61-72). Paris : L'Harmattan.

Charaudeau, P. (1983). Langage et discours. Éléments de sémiolinguistique (Théorie et pratique). Paris : Hachette.

Charaudeau, P. (2000). Le discours d'information médiatique: La construction du miroir social. Paris : Nathan.

De Certeau, M. (1990). L'invention du quotidien, tome 1 : Arts de faire. Paris : Gallimard. 
Détrez, C. (2004). Du quiproquo au monologue ? Rapports sexuels et rapports de sexe dans la littérature féminine contemporaine. Média et Information, Sexe et Communication, (20), 29-39.

Fleury, C. (2006). Imagination, imaginaire, imaginal. Paris : Presse Universitaires de France - PUF.

Freud, S. (2004). Introduction à la psychanalyse. Paris : Payot.

Goffman, E. (1977). La ritualisation de la féminité. Actes de la recherche en sciences sociales, 14(1), 34-50.

Goodman, N. (1992). Manières de faire des mondes. Nîmes : Chambon.

Heinich, N. (1996). États de femme. L'identité féminine dans la fiction occidentale. Paris : Gallimard.

Houdebine, A. (2004). Pour une sémiologie des indices (structurale et interprétative). Les cahiers du collège iconique, Communications et débats, (XVII), 148.

Kaufmann, J. (1995). Corps de femmes, regards d'hommes : sociologie des seins nus. Paris : Nathan.

Kaufmann, J. (2005). Le corps dans tous ses états : corps visible, corps sensible, corps secret. Dans C. Bromberger, Un corps pour soi (pp. 67-83). Paris: Presses Universitaires de France - PUF.

Monneyron, F. (2010). La photographie de mode - Un art souverain. Paris : Presses Universitaires de France - PUF.

Moscovici, S. (1976). Psychologie des représentations sociales. Cahiers Vilfredo Pareto, 14(38-39), 409-416.

Pahud, S. (2009). Variations publicitaires sur le genre : Une analyse linguistique des représentations publicitaires du féminin et du masculin. Zurich : Arttesia éditions.

Rivière, J. (1929). La féminité en tant que mascarade. International Journal of PsychoAnalysis, 10, 303-313.

Semprini, A. (2000). Analyser la communication : comment analyser les images, les médias, la publicité. Paris : L'Harmattan.

Soulages, J.-C. (2004). Le genre en publicité, ou le culte des apparences. MEI. Media et Information, (20), 51-59.

Soulages, J. (2009). Les avatars de la publicité télévisée ou les vies rêvées des femmes. Le Temps des médias, 12(1), 114-124.

Touraine, A. (2006). Le Monde des femmes. Paris : Fayard.

Veblen, T. B. (1978). Théorie de la classe de loisir (1899). Paris : Gallimard.

Veron, E. (1985). L'analyse du «contrat de lecture»: une nouvelle méthode pour les études de positionnement des supports de presse. Les médias, expériences, recherches actuelles, applications, IREP, 203-229.

Watzlawick, P. (1972). Une logique de la communication. Paris : Seuil. 